\title{
Structural abnormalities in the cuneus associated with Herpes Simplex Virus (type 1) infection in people at ultra high risk of developing psychosis
}

\section{Citation}

Whitford, Thomas J., Stephen J. Wood, Alison Yung, Luca Cocchi, Gregor Berger, Martha E. Shenton, Marek Kubicki, et al. 2012. Structural Abnormalities in the Cuneus Associated with Herpes Simplex Virus (type 1) Infection in People at Ultra High Risk of Developing Psychosis. Schizophrenia Research 135, no. 1-3: 175-180. doi:10.1016/j.schres.2011.11.003.

\section{Published Version}

doi:10.1016/j.schres.2011.11.003

\section{Permanent link}

http://nrs.harvard.edu/urn-3:HUL.InstRepos:28548932

\section{Terms of Use}

This article was downloaded from Harvard University's DASH repository, and is made available under the terms and conditions applicable to Other Posted Material, as set forth at http:// nrs.harvard.edu/urn-3:HUL.InstRepos:dash.current.terms-of-use\#LAA

\section{Share Your Story}

The Harvard community has made this article openly available.

Please share how this access benefits you. Submit a story.

Accessibility 


\title{
Structural abnormalities in the cuneus associated with Herpes Simplex Virus (type 1) infection in people at ultra high risk of developing psychosis
}

\author{
Thomas J. Whitford ${ }^{1,2, *}$, Stephen Wood ${ }^{1,3}$, Alison Yung ${ }^{4}$, Luca Cocchi ${ }^{1,5}$, Gregor Berger ${ }^{4}$, \\ Martha E. Shenton ${ }^{2}$, Marek Kubicki ${ }^{2}$, Lisa Phillips ${ }^{4}$, Dennis Velakoulis ${ }^{1}$, Robert H. Yolken ${ }^{6}$, \\ Christos Pantelis ${ }^{1}$, Patrick McGorry ${ }^{4}$, and G. Paul Amminger ${ }^{4,7}$ \\ ${ }^{1}$ Melbourne Neuropsychiatry Centre, Department of Psychiatry, University of Melbourne and \\ Melbourne Health, Australia \\ ${ }^{2}$ Psychiatry Neuroimaging Laboratory, Department of Psychiatry, Brigham and Women's Hospital, \\ Harvard Medical School, USA \\ ${ }^{3}$ School of Psychology, University of Birmingham, UK \\ ${ }^{4}$ ORYGEN Research Centre, University of Melbourne and Melbourne Health, Australia \\ ${ }^{5}$ Queensland Brain Institute, The University of Queensland, Australia \\ ${ }^{6}$ John Hopkins University School of Medicine, Stanley Division of Developmental Neurovirology, \\ Baltimore, Maryland, USA \\ ${ }^{7}$ Department of Child and Adolescent Psychiatry, Medical University, Vienna, Austria
}

\begin{abstract}
It has been suggested that some cases of schizophrenia may be caused by an interaction between physiological risk factors and exposure to certain neurotropic infectious agents such as Herpes Simplex Virus type 1 (HSV1). This study investigated whether HSV1 exposure was associated with structural brain abnormalities in individuals who, because of genetic or other factors, were deemed at ultra high risk (UHR) of developing psychosis. Twenty-five UHR individuals with a history of HSV1 exposure (HSV1+), 33 UHR participants without a history of HSV1 exposure (HSV1-) and 19 healthy controls participated in the study. All participants underwent a T1weighted structural MRI scan, and HSV1 exposure was determined based on the presence of IgG class antibodies in the blood serum. Voxel based morphometry revealed that the HSV1+ participants exhibited volumetric gray matter reductions in the cuneus, relative to both the HSV1and healthy control participants ( $<<0.05$, small volume corrected for familywise error). The results
\end{abstract}

(C) 2011 Elsevier B.V. All rights reserved.

*Corresponding author: Thomas J. Whitford, Melbourne Neuropsychiatry Centre, Department of Psychiatry, The University of Melbourne, National Neuroscience Facility, Level 3, Alan Gilbert Building, 161 Barry Street, Carlton South, VIC, 3053, Australia, Phone: +613 83441861, wht@unimelb.edu.au.

Contributors

TW was involved in data processing and analysis, and wrote the first draft of the manuscript. SW, AY, LC, GB, LP, DV, CP, PM, PA were involved in participant recruitment, data acquisition, processing and interpretation. MS, MK, RY were involved in were involved in data analysis, processing and interpretation. All authors contributed to and have approved the final manuscript.

Conflict of Interest

All authors declare that they have no conflicts of interest.

Publisher's Disclaimer: This is a PDF file of an unedited manuscript that has been accepted for publication. As a service to our customers we are providing this early version of the manuscript. The manuscript will undergo copyediting, typesetting, and review of the resulting proof before it is published in its final citable form. Please note that during the production process errors may be discovered which could affect the content, and all legal disclaimers that apply to the journal pertain. 
of the study suggest that a history of HSV1 infection is associated with volumetric gray matter reductions in individuals at ultra-high risk for developing psychosis, and are consistent with previous studies that have identified structural gray matter abnormalities in HSV1-infected patients with established schizophrenia.

\section{Keywords}

schizophrenia; viral load; infectious agents; Magnetic Resonance Imaging; voxel-based morphometry

\section{Introduction}

It has long been suggested that the development of schizophrenia may in some cases be associated with exposure to infectious agents (Crow, 1984; O'Reilly, 1994; Pearce, 2001; Torrey et al., 2006; Yolken et al., 2009; Yolken and Torrey, 1995, 2008). Support for this hypothesis comes from the fact that psychotic individuals are uncommonly likely to have been born during periods of elevated infectious agent activity (Mednick et al., 1988; Torrey et al., 1988), and that babies born in winter, which is the period in which transmissible infections are most likely to occur, are more likely to subsequently develop schizophrenia than babies born in other seasons (Mortensen et al., 1999; Torrey et al., 1997) - see Brown and Derkits (2010) for a review. The Herpes Simplex Virus Type 1 has been specifically proposed as a contributing factor in a minority of cases of schizophrenia (Yolken, 2004). Herpes Simplex Virus Type 1 (HSV1) is a common virus that has an estimated, age-related seroprevalance of between 30 and $80 \%$ in developed countries (Cunningham et al., 2006; Smith and Robinson, 2002). HSV1 is commonly associated with the development of oral and facial lesions ('cold sores') in infected individuals. HSV1 is a neurotropic virus that has the ability to lie dormant in infected neurons - often in the trigeminal ganglia (Croen et al., 1987) - for several years before reactivating. The fact that HSV1 preferentially infects nervous cells and is capable of replicating in the central nervous system has led to it being implicated as a potential environmental trigger in a minority of schizophrenia cases (Yolken, 2004). While the mechanisms of pathogenesis remain a matter of debate, one possibility is that the cycles of latency and reactivation characteristic of HSV1 could cause structural damage to the host cells, which could consequently lead to schizophrenia in predisposed individuals (Pearce, 2001).

Two recent studies provide support for the idea that HSV1 infection could be associated with structural damage to central nervous system cells in patients with schizophrenia. Prasad et al. (2007) used voxel-based morphometry and identified volumetric gray matter reductions in the dorsolateral prefrontal cortex and anterior cingulate gyrus in first-episode schizophrenia patients with a history of HSV1 exposure, relative to uninfected first-episode schizophrenia participants. Interestingly, however, Prasad et al. (2007) did not observe an association between HSV1 exposure and brain structure in healthy individuals. They suggested that this result could reflect an interaction between HSV1 exposure and some biologically-based risk factor(s) associated with schizophrenia (e.g., certain genetic variants), such that only those individuals in possession of this risk factor would develop structural brain abnormalities in response to HSV1 infection. This hypothesis was supported by a subsequent voxel-based morphometry study by Schretlen et al. (2010), who found that patients with chronic schizophrenia who were seropositive for HSV1 showed volumetric GM reductions in the anterior cingulate gyrus, cerebellum, cuneus and thalamus, relative to seronegative schizophrenia patients. Thus there is some evidence to suggest that exposure to HSV1 can result in structural brain abnormalities in patients with established schizophrenia. 
The evidence for an association between HSV1 exposure and brain structure in patients with established schizophrenia raises the question of whether individuals who do not have schizophrenia, but who are at high risk of developing a psychotic disorder (and who thus may be more likely to possess risk factors for schizophrenia) also show this association. This question has not (to our knowledge) been investigated previously. The present study investigated this question by using voxel-based morphometry to compare the brain structure of people deemed at "ultra-high risk" (UHR) of developing a psychotic disorder, who also had a history of HSV1 infection (based on the presence of serum immunoglobulin G antibodies), with UHR individuals without a history of HSV1 infection, and matched healthy controls. Based on the aforementioned results of Prasad et al. (2007) and Schretlen et al. (2010), who both found HSV1 exposure to be associated with volumetric gray matter reductions in patients with established schizophrenia, we hypothesized that the HSV1 seropositive UHR participants would exhibit volumetric grey matter reductions in the anterior cingulate gyrus, dorsolateral prefrontal cortex, thalamus, cuneus and/or cerebellum, relative to the HSV1 seronegative UHR participants, and matched healthy controls.

\section{Methods}

\subsection{Setting}

The study was conducted at the Personal Assessment and Crisis Evaluation (PACE) clinic, Orygen Youth Health, Melbourne, Australia. The PACE clinic is a clinical service for young people at ultra high risk (UHR) of developing a psychotic disorder. Referrals to the PACE Clinic came from general practitioners, teachers and university health services, drug and alcohol services, youth support organizations such as homeless services, and other mental health services, including the wider Orygen Youth Health service (McGorry et al., 2002; Yung et al., 1998).

\subsection{UHR Criteria}

The intake criteria for the UHR participants were (a) age of between 14 and 30 years (b) residence in the Melbourne metropolitan area and (c) at least one of the following criteria for UHR status: (1) Attenuated Psychotic Symptoms: presence of attenuated (sub-threshold) psychotic symptoms within the previous 12 months. (2) Brief Limited Intermittent Psychotic Symptoms: history of brief, self limited psychotic symptoms that had spontaneously resolved within the previous 12 months. (3) Trait Group: presumed genetic vulnerability to psychotic disorder (i.e., either schizotypal personality disorder or family history of psychotic disorder in a first degree relative) plus persistent low functioning for at least one month within the previous 12 months. These UHR criteria have been described in more detail previously (Yung et al., 2003; Yung et al., 2004). The UHR intake criteria were assessed with the Comprehensive Assessment of At Risk Mental States (CAARMS) (Yung et al., 2005).

\subsection{Participants}

Fifty-eight UHR participants were recruited for the study. These participants were a subset of the participant sample described in Amminger et al. (2007). These participants met the following criteria for UHR status: Attenuated Psychotic Symptoms $=45$ of 58 (77.6\%), Brief, Limited Intermittent Psychotic Symptoms $=6$ of 58 (10.3\%), Trait Group $=19$ of 58 (32.7\%). The HSV1+ and HSV1-participants were compared to a group of 19 healthy control (HC) participants. The HC participants were recruited from similar sociodemographic areas as the UHR participants by approaching ancillary hospital staff, and through advertisements. The demographic and clinical details for the three groups are presented in Table 1 . The three groups did not differ significantly from each other in terms of age, sex, handedness or premorbid IQ, which was measured with the National Adult 
Reading Test (NART) (Nelson, 1982). Furthermore, the two patient groups (i.e., HSV1+ and HSV1-) did not significantly differ from each other in terms of their score on the Psychosis subscale of the Brief Psychiatric Rating Scale (BPRS) (Overall and Gorham, 1962), global score on Scale for the Assessment of Negative Symptoms (SANS) (Andreasen, 1984) or Global Assessment of Functioning (GAF) score (Hall, 1995). The 58 UHR individuals who participated in the study were recruited between April 2001 and December 2004. Of these participants, 7 had transitioned to a psychotic disorder as defined by the CAARMS by July 2010.

Exclusion criteria for all participants were: 1) known history of a previous psychotic or manic episode (treated or untreated); 2) history of substantial head injury, seizures, neurological disorders, impaired thyroid function, corticosteroid use; 3 ) lifetime antipsychotic dose of $15 \mathrm{mg}$ of haloperidol (or equivalent) or greater; 3) any previous or current use of mood stabilizing medication; 4) intellectual disability (IQ < 70). All participants reported English as their preferred language. The study was approved by the North-Western Mental Health Program Research and Ethics Committee, Melbourne, Australia. All participants provided written informed consent, as well as parental consent for those participants less than 18 years of age.

\subsection{Immunological Assays}

Blood samples were taken from all participants within three weeks of acceptance to the PACE clinic. Serum IgG class antibodies were measured to HSV1 using previously described immunoassay methods (Buka et al., 2001; Yolken et al., 2001). The assay was defined as IgG seropositive or seronegative based on comparison with the reactivity of specific antibody standards assayed along with the blood samples (Sauerbrei and Wutzler, 2004). All serological tests were carried out at the Stanley Laboratory of Developmental Neurovirology, Baltimore, Maryland.

\subsection{Magnetic Resonance Image Acquisition and Pre-Processing}

The protocol used for MRI acquisition has been described previously (Wood et al., 2010). All scanning was performed using a 3 T GE LX Horizon scanner (GE Healthcare, Milwaukee, USA) at the Brain Research Institute, Victoria. A 3-dimensional volumetric spoiled gradient recalled echo in the steady state sequence generated 124 contiguous coronal slices. Imaging parameters were as follows: time to echo $=9 \mathrm{~ms}$; time to repetition $=36 \mathrm{~ms}$; flip angle $=30^{\circ}$; matrix size $=410 \times 410$; field of view $=20 \times 20 \mathrm{~cm}$ matrix; voxel dimension $=0.4883 \times 0.4883 \times 2 \mathrm{~mm}$

The MR images were processed using voxel-based morphometry (VBM) in SPM2 (Wellcome Department of Cognitive Neurology, London, UK), running on Matlab 6.5 (MathWorks, Natick, USA). The protocol for pre-processing has been described in detail elsewhere (Whitford et al., 2006). The raw MR images were first spatially normalized to a customized template, (ICBM 152 template), using a 12-parameter affine transformation and a non-linear warp (Ashburner and Friston, 2000). The normalized images were re-sliced with $2 \mathrm{~mm}^{3}$ voxels, and segmented into grey matter, white matter, and cerebrospinal fluid probability images on the basis of signal intensity and an a priori expectation of the anatomical location of the different tissue classes. The grey matter images were then modulated with the Jacobian determinants derived from the spatial normalization (Good et al., 2001) and smoothed with a $12 \mathrm{~mm}$ Gaussian kernel.

\subsection{Statistical Analysis}

The UHR participants were divided into HSV1 seropositive (HSV1+) and HSV1 seronegative (HSV1-) groups, and compared with a group of matched healthy controls (HC). 
An initial, exploratory whole-brain analysis-of-variance (ANOVA) was performed to investigate for voxelwise differences between the HSV1+, HSV1- and HC groups in the grey matter volume of every voxel in the processed images. For this exploratory analysis, a was set to 0.05 and was corrected for familywise error across every voxel in the processed grey matter images (i.e., whole-brain corrected for familywise error). The primary analysis involved an ANOVA in the voxels defined by five regions-of-interest (ROIs), namely the anterior cingulate gyrus, dorsolateral prefrontal cortex, cerebellum, cuneus and thalamus. These five ROIs were chosen on the basis that they were the regions where HSV1 related differences in grey matter volume were observed by Prasad et al. (2007) and Schretlen et al. (2010) in patients with established schizophrenia. The five ROIs - illustrated in Figure 1 were created from the Automatic Anatomical Labeling (AAL) masks (Tzourio-Mazoyer et al., 2002). The dorsolateral prefrontal cortex ROI was created by convolving the "superior frontal gyrus" and "middle frontal gyrus" AAL masks. The cerebellum ROI was taken by convolving the "cerebellum 3, 4_5, 6, 7b, 8, 9, 10, crus1 and crus2" AAL masks. The anterior cingulate, cuneus, and thalamus ROIs were taken directly from their respective AAL masks. For the ROI analysis, a was set to 0.05 , and was corrected for familywise error across the voxels defined by the ROI (i.e., small-volume corrected for familywise error).

\section{Results}

Of the 58 UHR individuals who participated in the study, 25 (43.1\%) were classified as being IgG seropositive to HSV1 on the basis of the immunological assay run on their blood sample (HSV1+), while 33 UHR participants (56.9\%) were classified as being IgG seronegative (HSV1-).

For the exploratory whole-brain analysis, univariate ANOVA revealed no significant differences in voxel-wise grey matter volume between the HSV1+, HSV1- and HC groups $(\mathrm{p}<0.05$, corrected for familywise error over all gray matter voxels). In contrast, the ROI analysis revealed a significant between-group difference in the volume of a cluster of 22 voxels in the cuneus ROI (MNI coordinate [-12 -74 30], $\mathrm{F}_{2,77}=8.26, \mathrm{p}=0.038$, small-volume corrected for familywise error). Post hoc t-tests revealed that the significant ANOVA was driven by the HSV1+ group exhibiting grey matter reductions in the cuneus, relative to both the HC (see Table 2 and Fig 2a) and HSV1- groups (see Table 2 and Fig 2b). All results remained statistically significant when age and gender were entered as nuisance covariates.

The results of the voxelwise analyses were confirmed in a supplementary analysis, in which the cuneus ROI was extracted from each participant's grey matter image (as per the protocol of Whitford et al., 2009), and its volume compared between the three clinical groups. Univariate ANOVA (Bonferroni corrected for multiple comparisons across the 5 ROIs) revealed a significant difference in the volume of the cuneus between the three groups $\left(\mathrm{F}_{2,77}=5.491, \mathrm{p}=0.006\right.$, eta $\left.^{2}=0.129\right)$. Consistent with the voxelwise results, post-hoc (Tukey) contrasts confirmed that the significant ANOVA was driven by the HSV1+ group exhibiting significantly reduced cuneus volumes relative to both the HC group (mean difference $=$ 0.859 , standard error $=0.285, \mathrm{p}=0.010$; see Figure 2c), and the HSV1- group (mean difference $=0.6715$, standard error $=0.2482, p=0.023$; see Figure $2 \mathrm{~d}$ ). There was no significant difference in cuneus volume between the HC and HSV1- groups (mean difference $=0.1844$, standard error $=0.269, \mathrm{p}=0.774$ ).

$8.0 \%$ ( 2 out of 25 ) of the HSV1+ participants, and $15.1 \%$ (5 out of 33) of the HSVparticipants subsequently transitioned to psychosis. The proportion of UHR participants who transitioned to psychosis was not significantly between the HSV1+ and HSV1- groups (Pearson Chi-squared=0.686, $\mathrm{p}=0.408$; Likelihood Ratio $=0.712, \mathrm{p}=.399$; Goodman and Kruskal Tau $=0.012, \mathrm{p}=.412$ ). 


\section{Discussion}

The present study found that participants at ultra-high risk (UHR) of developing a psychotic disorder, who had a history of HSV1 infection, exhibited grey matter reductions in the cuneus relative to both UHR participants without a history of HSV1 infection, and matched healthy controls. This result extends the results of previous studies that have identified structural gray matter reductions in HSV1 infected patients with established schizophrenia, relative to uninfected patients (Prasad et al., 2007; Schretlen et al., 2010), including in the cuneus (Schretlen et al., 2010).

While structural abnormalities in the cuneus have previously been reported in both chronic and first-episode patients with schizophrenia (Cascella et al., 2010; Cocchi et al., 2009; Tanskanen et al., 2010; Whitford et al., 2006), this is the first study (to our knowledge) that has identified cuneus abnormalities in UHR individuals; i.e., UHR participants with a history of HSV1 infection showed gray matter abnormalities in the cuneus relative to the matched healthy controls. Although the cuneus is believed to have a variety of cognitive functions - including in working memory (Bluhm et al., 2010), cognitive control (Haldane et al., 2008) and behavioral engagement (Zhang and Li, 2011) - it is primarily believed to be involved in visual processing. In particular, there is evidence that the cuneus is involved in monitoring changes in ocular position in response to self-generated eye movements (Law et al., 1998). In light of this, it is interesting to note that abnormalities in smooth pursuit eye movements have consistently been reported in patients with schizophrenia (Levy et al., 1994), and have also been observed in UHR participants (van Tricht et al., 2010). Given that smooth pursuit eye movements are highly dependent on the accurate monitoring of eye position, these findings suggest that people who either have schizophrenia, or who are at risk for developing the disorder, may have abnormalities in monitoring their self-generated eye movements. This may be indicative of a more general self-monitoring abnormality, such as has been suggested to underlie many of the psychotic symptoms of schizophrenia (Feinberg, 1978; Frith, 1992). The question of whether the structural abnormalities in the cuneus exhibited by the HSV1+ participants in the present study are also associated with eye tracking abnormalities is a worthwhile topic for future investigation.

A significant strength of the study is the fact that the UHR participants were neuroleptic naïve. This fact is significant in light of growing evidence that neuroleptic medications can influence brain structure in and of themselves (Ho et al., 2011; Konopaske et al., 2008), which makes it difficult to disentangle the brain changes associated with psychosis from the brain changes associated with neuroleptic exposure. However, a limitation of the study is that blood samples were not acquired from the control group. This is problematic as it meant that HSV1 serostatus could not be ascertained for the control participants, thus raising the possibility that HSV1 exposure could have been associated with cuneus abnormalities in both the control and UHR participants. While this possibility is inconsistent with the findings of Prasad et al. (2007), who did not observe a significant association between the gray matter volume of any voxel and HSV1 serostatus in healthy individuals, it would nonetheless be advantageous to replicate this in an independent control sample. The lack of a relationship between HSV1 serostatus and the rate of transition to full-blown psychosis also raises a question as to the clinical relevance of the observed gray matter reductions in the HSV1+ participants. However, the fact that only 7 of the 58 UHR participants subsequently transitioned to psychosis meant that the analysis of HSV1 serostatus vs. psychosis transition rate was severely underpowered and thus the results of this analysis should be considered with the utmost caution. Notwithstanding the fact that our criteria for UHR status are among the most well-validated and widely-used in the neuropsychiatric literature, it should also be acknowledged that the low transition rate raises the question as to whether our sample was truly representative of an "ultra-high risk" population. 
In summary, this is the first study to investigate the relationship between brain structure and HSV1 exposure in individuals at ultra-high risk of developing psychosis. The results revealed that UHR participants with a history of HSV1 infection exhibited volumetric grey matter reductions in the cuneus, relative to both UHR participants without a history of HSV1 infection, and matched healthy controls. This result extends the results of previous studies that have identified structural gray matter reductions in HSV1 infected patients with established schizophrenia relative to uninfected patients (Prasad et al., 2007; Schretlen et al., 2010).

\section{Acknowledgments}

\section{Role of Funding Source}

This work was supported by the National Health and Medical Research Council of Australia (Overseas-Based Postdoctoral Training Fellowship 520627 to T.J.W Program Grant 145627; Program Grant 350421 and 566529; Clinical Career Development Awards to S.W, A.Y, C.P); NARSAD Brain and Behavior Research Foundation (Young Investigator Award) to T.J.W and S.W.; the National Institutes of Health (R03 MH068464-0 to M.K.; R01 MH 50747, K05 MH 070047, and P50 MH 080272 to M.E.S.); Harvard Medical School (Milton Award); Department of Veterans Affairs (VA Merit Award, VA Research Enhancement Award Program, and VA Schizophrenia Research Center Grant to M.E.S.; Stanley Medical Research Institute to R.H.Y.

The authors would like to thank participants of the study.

\section{References}

Amminger G, McGorry P, Berger G, Wade D, Yung A, Phillips L, Harrigan S, Francey S, Yolken R. Antibodies to infectious agents in individuals at ultra-high risk for psychosis. Biol Psychiatry. 2007; 61:1215-1217. [PubMed: 17207471]

Andreasen, NC. Scale for the Assessment of Negative Symptoms (SANS). University of Iowa; Iowa City: 1984.

Ashburner J, Friston KJ. Voxel-based morphometry--the methods. Neuroimage. 2000; 11:805-821. [PubMed: 10860804]

Bluhm RL, Clark CR, McFarlane AC, Moores KA, Shaw ME, Lanius RA. Default network connectivity during a working memory task. Hum Brain Mapp. 2010

Brown AS, Derkits EJ. Prenatal infection and schizophrenia: a review of epidemiologic and translational studies. Am J Psychiatry. 2010; 167:261-280. [PubMed: 20123911]

Buka S, Tsuang M, Torrey E, Klebanoff M, Bernstein D, Yolken R. Maternal infections and subsequent psychosis among offspring. Arch Gen Psychiatry. 2001; 58:1032-1037. [PubMed: 11695949]

Cascella NG, Fieldstone SC, Rao VA, Pearlson GD, Sawa A, Schretlen DJ. Gray-matter abnormalities in deficit schizophrenia. Schizophr Res. 2010; 120:63-70. [PubMed: 20452187]

Cocchi L, Walterfang M, Testa R, Wood SJ, Seal ML, Suckling J, Takahashi T, Proffitt TM, Brewer WJ, Adamson C, Soulsby B, Velakoulis D, McGorry PD, Pantelis C. Grey and white matter abnormalities are associated with impaired spatial working memory ability in first-episode schizophrenia. Schizophr Res. 2009; 115:163-172. [PubMed: 19837566]

Croen KD, Ostrove JM, Dragovic LJ, Smialek JE, Straus SE. Latent herpes simplex virus in human trigeminal ganglia. Detection of an immediate early gene "anti-sense" transcript by in situ hybridization. N Engl J Med. 1987; 317:1427-1432. [PubMed: 2825014]

Crow T. A re-evaluation of the viral hypothesis: is psychosis the result of retroviral integration at a site close to the cerebral dominance gene? Br J Psychiatry. 1984; 145:243-253. [PubMed: 6089945]

Cunningham AL, Taylor R, Taylor J, Marks C, Shaw J, Mindel A. Prevalence of infection with herpes simplex virus types 1 and 2 in Australia: a nationwide population based survey. Sex Transm Infect. 2006; 82:164-168. [PubMed: 16581748]

Feinberg I. Efference copy and corollary discharge: implications for thinking and its disorders. Schizophr Bull. 1978; 4:636-640. [PubMed: 734369] 
Frith, CD. The cognitive neuropsychology of schizophrenia. Lawrence Erlbaum Associates; Hove, UK: 1992.

Good CD, Johnsrude I, Ashburner J, Henson RNA, Friston KJ, Frackowiak RSJ. A voxel-based morphometric study of aging in 465 normal adult human brains. NeuroImage. 2001; 14:21-36. [PubMed: 11525331]

Haldane M, Cunningham G, Androutsos C, Frangou S. Structural brain correlates of response inhibition in Bipolar Disorder I. J Psychopharmacol. 2008; 22:138-143. [PubMed: 18308812]

Hall RC. Global assessment of functioning. A modified scale. Psychosomatics. 1995; 36:267-275. [PubMed: 7638314]

Ho BC, Andreasen NC, Ziebell S, Pierson R, Magnotta V. Long-term antipsychotic treatment and brain volumes: a longitudinal study of first-episode schizophrenia. Arch Gen Psychiatry. 2011; 68:128-137. [PubMed: 21300943]

Konopaske G, Dorph-Petersen K, Sweet R, Pierri J, Zhang W, Sampson A, Lewis D. Effect of chronic antipsychotic exposure on astrocyte and oligodendrocyte numbers in macaque monkeys. Biol Psychiatry. 2008; 63:759-765. [PubMed: 17945195]

Law I, Svarer C, Rostrup E, Paulson OB. Parieto-occipital cortex activation during self-generated eye movements in the dark. Brain. 1998; 121(Pt 11):2189-2200. [PubMed: 9827777]

Levy DL, Holzman PS, Matthysse S, Mendell NR. Eye tracking and schizophrenia: a selective review. Schizophr Bull. 1994; 20:47-62. [PubMed: 8197421]

McGorry P, Yung A, Phillips L, Yuen H, Francey S, Cosgrave E, Germano D, Bravin J, McDonald T, Blair A, Adlard S, Jackson H. Randomized controlled trial of interventions designed to reduce the risk of progression to first-episode psychosis in a clinical sample with subthreshold symptoms. Arch Gen Psychiatry. 2002; 59:921-928. [PubMed: 12365879]

Mednick SA, Machon RA, Huttunen MO, Bonett D. Adult schizophrenia following prenatal exposure to an influenza epidemic. Arch Gen Psychiatry. 1988; 45:189-192. [PubMed: 3337616]

Mortensen PB, Pedersen CB, Westergaard T, Wohlfahrt J, Ewald H, Mors O, Andersen PK, Melbye M. Effects of family history and place and season of birth on the risk of schizophrenia. N Engl J Med. 1999; 340:603-608. [PubMed: 10029644]

Nelson H. The National Adult Reading Test (NART): test manual. 1982

O’Reilly R. Viruses and schizophrenia. Aust N Z J Psychiatry. 1994; 28:222-228. [PubMed: 7527632]

Overall JE, Gorham DR. The Brief Psychiatric Rating Scale. Psychological Reports. 1962; 10:799_ 812.

Pearce B. Schizophrenia and viral infection during neurodevelopment: a focus on mechanisms. Mol Psychiatry. 2001; 6:634-646. [PubMed: 11673791]

Prasad K, Shirts B, Yolken R, Keshavan M, Nimgaonkar V. Brain morphological changes associated with exposure to HSV1 in first-episode schizophrenia. Mol Psychiatry. 2007; 12:105-113. 101. [PubMed: 17033628]

Sauerbrei A, Wutzler P. Serological detection of type-specific IgG to herpes simplex virus by novel ELISAs based on recombinant and highly purified glycoprotein G. Clin Lab. 2004; 50:425-429. [PubMed: 15330511]

Schretlen DJ, Vannorsdall TD, Winicki JM, Mushtaq Y, Hikida T, Sawa A, Yolken RH, Dickerson FB, Cascella NG. Neuroanatomic and cognitive abnormalities related to herpes simplex virus type 1 in schizophrenia. Schizophr Res. 2010; 118:224-231. [PubMed: 20153952]

Smith JS, Robinson NJ. Age-specific prevalence of infection with herpes simplex virus types 2 and 1: a global review. J Infect Dis. 2002; 186(Suppl 1):S3-28. [PubMed: 12353183]

Tanskanen P, Ridler K, Murray GK, Haapea M, Veijola JM, Jaaskelainen E, Miettunen J, Jones PB, Bullmore ET, Isohanni MK. Morphometric brain abnormalities in schizophrenia in a populationbased sample: relationship to duration of illness. Schizophr Bull. 2010; 36:766-777. [PubMed: 19015212]

Torrey E, Leweke M, Schwarz M, Mueller N, Bachmann S, Schroeder J, Dickerson F, Yolken R. Cytomegalovirus and schizophrenia. CNS Drugs. 2006; 20:879-885. [PubMed: 17044725]

Torrey EF, Miller J, Rawlings R, Yolken RH. Seasonality of births in schizophrenia and bipolar disorder: a review of the literature. Schizophr Res. 1997; 28:1-38. [PubMed: 9428062] 
Torrey EF, Rawlings R, Waldman IN. Schizophrenic births and viral diseases in two states. Schizophr Res. 1988; 1:73-77. [PubMed: 3154510]

Tzourio-Mazoyer N, Landeau B, Papathanassiou D, Crivello F, Etard O, Delcroix N, Mazoyer B, Joliot M. Automated anatomical labeling of activations in SPM using a macroscopic anatomical parcellation of the MNI MRI single-subject brain. Neuroimage. 2002; 15:273-289. [PubMed: 11771995]

van Tricht MJ, Nieman DH, Bour LJ, Boeree T, Koelman JH, de Haan L, Linszen DH. Increased saccadic rate during smooth pursuit eye movements in patients at Ultra High Risk for developing a psychosis. Brain Cogn. 2010; 73:215-221. [PubMed: 20538400]

Whitford TJ, Farrow TF, Williams LM, Gomes L, Brennan J, Harris AW. Delusions and dorso-medial frontal cortex volume in first-episode schizophrenia: a voxel-based morphometry study. Psychiatry Res. 2009; 172:175-179. [PubMed: 19395244]

Whitford TJ, Grieve SM, Farrow TF, Gomes L, Brennan J, Harris AWF, Gordon E, Williams LM. Progressive grey matter atrophy over the first 2-3 years of illness in first-episode schizophrenia: a tensor-based morphometry study. NeuroImage. 2006; 32:511-519. [PubMed: 16677830]

Wood SJ, Kennedy D, Phillips LJ, Seal ML, Yucel M, Nelson B, Yung AR, Jackson G, McGorry PD, Velakoulis D, Pantelis C. Hippocampal pathology in individuals at ultra-high risk for psychosis: a multi-modal magnetic resonance study. NeuroImage. 2010; 52:62-68. [PubMed: 20399273]

Yolken R. Viruses and schizophrenia: a focus on herpes simplex virus. Herpes. 2004; 11(Suppl 2): 83A-88A.

Yolken R, Bachmann S, Ruslanova I, Lillehoj E, Ford G, Torrey E, Schroeder J, Rouslanova I. Antibodies to Toxoplasma gondii in individuals with first-episode schizophrenia. Clin Infect Dis. 2001; 32:842-844. [PubMed: 11229859]

Yolken R, Dickerson F, Fuller Torrey E. Toxoplasma and schizophrenia. Parasite Immunol. 2009; 31:706-715. [PubMed: 19825110]

Yolken R, Torrey E. Viruses, schizophrenia, and bipolar disorder. Clin Microbiol Rev. 1995; 8:131145. [PubMed: 7704891]

Yolken R, Torrey E. Are some cases of psychosis caused by microbial agents? A review of the evidence. Mol Psychiatry. 2008; 13:470-479. [PubMed: 18268502]

Yung A, Phillips L, McGorry P, McFarlane C, Francey S, Harrigan S, Patton G, Jackson H. Prediction of psychosis. A step towards indicated prevention of schizophrenia. Br J Psychiatry Suppl. 1998; 172:14-20. [PubMed: 9764121]

Yung A, Phillips L, Yuen H, Francey S, McFarlane C, Hallgren M, McGorry P. Psychosis prediction: 12-month follow up of a high-risk ("prodromal”) group. Schizophr Res. 2003; 60:21-32. [PubMed: 12505135]

Yung AR, Phillips LJ, Yuen HP, McGorry PD. Risk factors for psychosis in an ultra high-risk group: psychopathology and clinical features. Schizophr Res. 2004; 67:131-142. [PubMed: 14984872]

Yung AR, Yuen HP, McGorry PD, Phillips LJ, Kelly D, Dell'Olio M, Francey SM, Cosgrave EM, Killackey E, Stanford C, Godfrey K, Buckby J. Mapping the onset of psychosis: the Comprehensive Assessment of At-Risk Mental States. Aust N Z J Psychiatry. 2005; 39:964-971. [PubMed: 16343296]

Zhang S, Li CS. Functional networks for cognitive control in a stop signal task: Independent component analysis. Hum Brain Mapp. 2011 


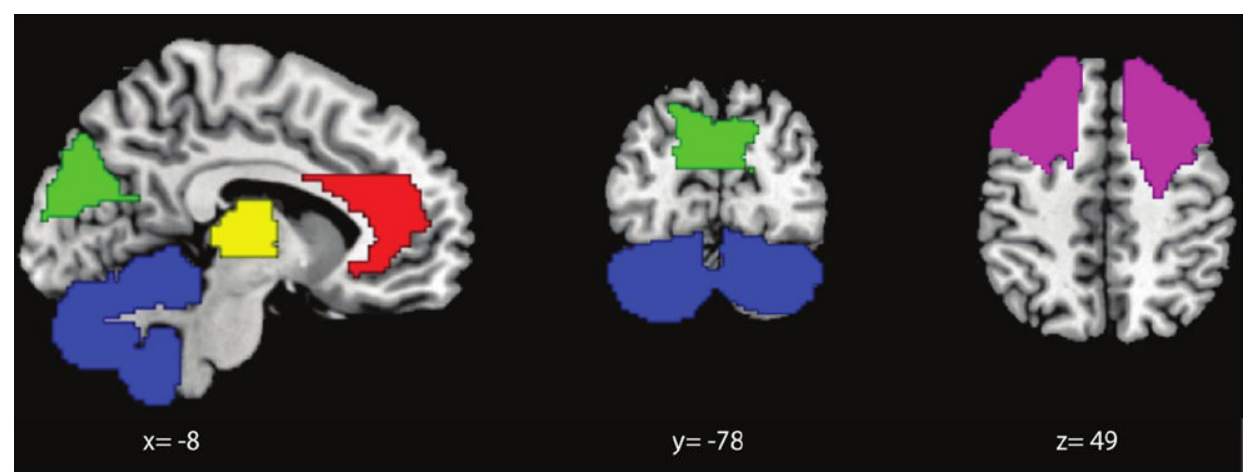

Figure 1.

The five regions-of-interest (ROIs) in which the gray matter volumes of the constituent voxels were compared between the three clinical groups (HSV1+, HSV1- and HC). The five ROIs were: anterior cingulate gyrus (red), dorsolateral prefrontal cortex (purple), cerebellum (blue), cuneus (green), and thalamus (yellow). The ROIs were created from the Automatic Anatomical Labeling (AAL) masks (see Methods), and were chosen on the basis that they were the regions where HSV1 related differences in grey matter volume were observed by Prasad et al. (2007) and Schretelen et al. (2010) in patients with established schizophrenia. The ROIs are presented overlaid onto slices of a T1-weighted MR image in MNI space 


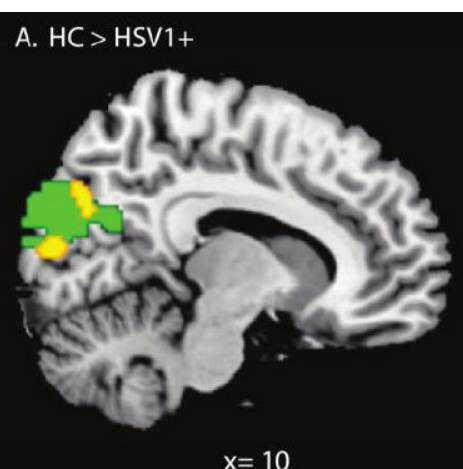

$x=10$
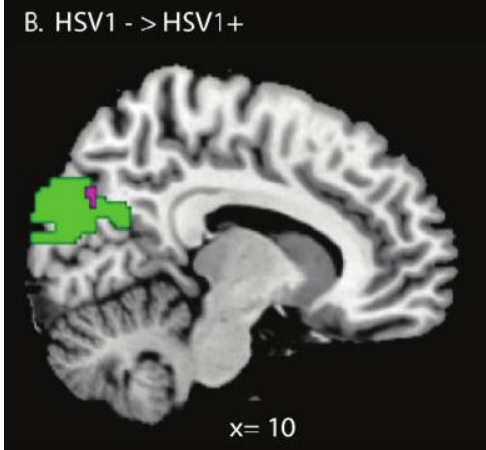
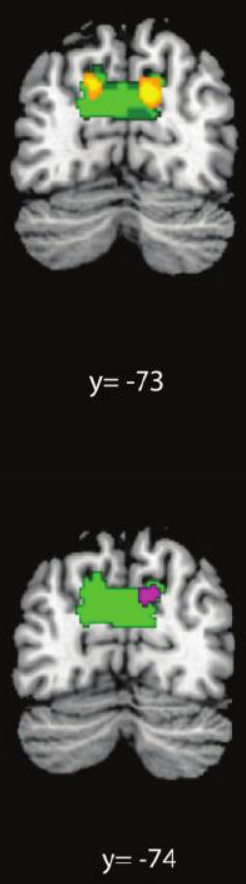

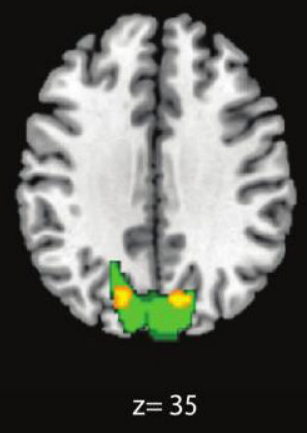

C.

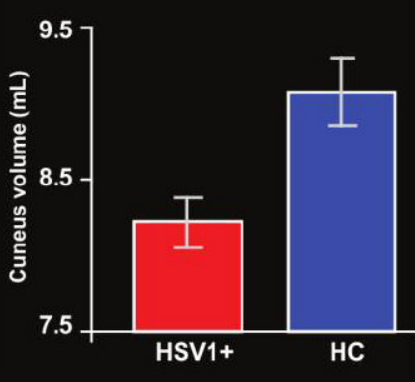

D.

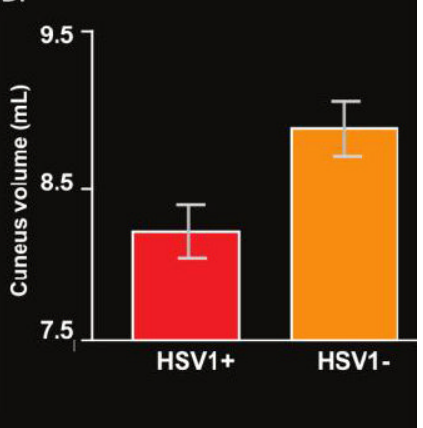

Figure 2.

Voxels within the Cuneus ROI for which there were differences in gray matter volume between the three clinical groups (HSV1+, HSV1- and HC). Panel A depicts the voxels (in yellow) in the Cuneus ROI (in green) where the HSV1+ patients exhibited volumetric gray matter reductions relative to the $\mathrm{HC}$ participants $(\mathrm{p}<0.05$, small-volume corrected for familywise error). Panel B depicts the voxels (in purple) in the Cuneus ROI (in green) where the HSV1+ patients exhibited volumetric gray matter reductions relative to the HSV1patients ( $p<0.05$, small-volume corrected for familywise error, Left/Right inverted). The clusters are presented in radiological space (left/right inverted) and overlaid on slices from a T1-weighted MR image in MNI space. Panel C depicts the difference between the HSV1+ and HC groups in terms of the total gray matter volume $(\mathrm{mL})$ of the extracted Cuneus ROI. Panel D depicts the difference between the HSV1+ and HSV1- groups in terms of the total gray matter volume $(\mathrm{mL})$ of the extracted Cuneus ROI. 
Table 1

Demographic and clinical details for the three clinical groups

\begin{tabular}{lcccc}
\hline & HSVI- $(\boldsymbol{n}=\mathbf{3 3})$ & $\boldsymbol{H S V I +}(\boldsymbol{n}=\mathbf{2 5})$ & $\boldsymbol{H C}(\boldsymbol{n = 1 9 )}$ & $\boldsymbol{P}$-value (ANOVA) \\
\hline Age & $18.56(3.28)$ & $20.31(3.40)$ & $20.98(5.02)$ & $\mathrm{P}=.06$ \\
Sex & $15 \mathrm{~F} / 18 \mathrm{M}$ & $11 \mathrm{~F} / 14 \mathrm{M}$ & $12 \mathrm{~F} / 7 \mathrm{M}$ & $\mathrm{P}=.38$ \\
Hand & $31 \mathrm{R} / 2 \mathrm{~L}$ & $24 \mathrm{R} / 1 \mathrm{~L}$ & $19 \mathrm{R} / 0 \mathrm{~L}$ & $\mathrm{P}=.55$ \\
Premorbid IQ & $100.00(12.54)$ & $101.52(10.99)$ & $101.93(11.70)$ & $\mathrm{P}=.84$ \\
BPRS (Psychosis) & $6.48(3.14)$ & $6.52(2.54)$ & - & $\mathrm{P}=.96$ \\
SANS-Total & $28.91(15.56)$ & $31.28(18.15)$ & - & $\mathrm{P}=.59$ \\
GAF & $56.94(8.36)$ & $53.36(15.52)$ & - & $\mathrm{P}=.67$ \\
\hline
\end{tabular}




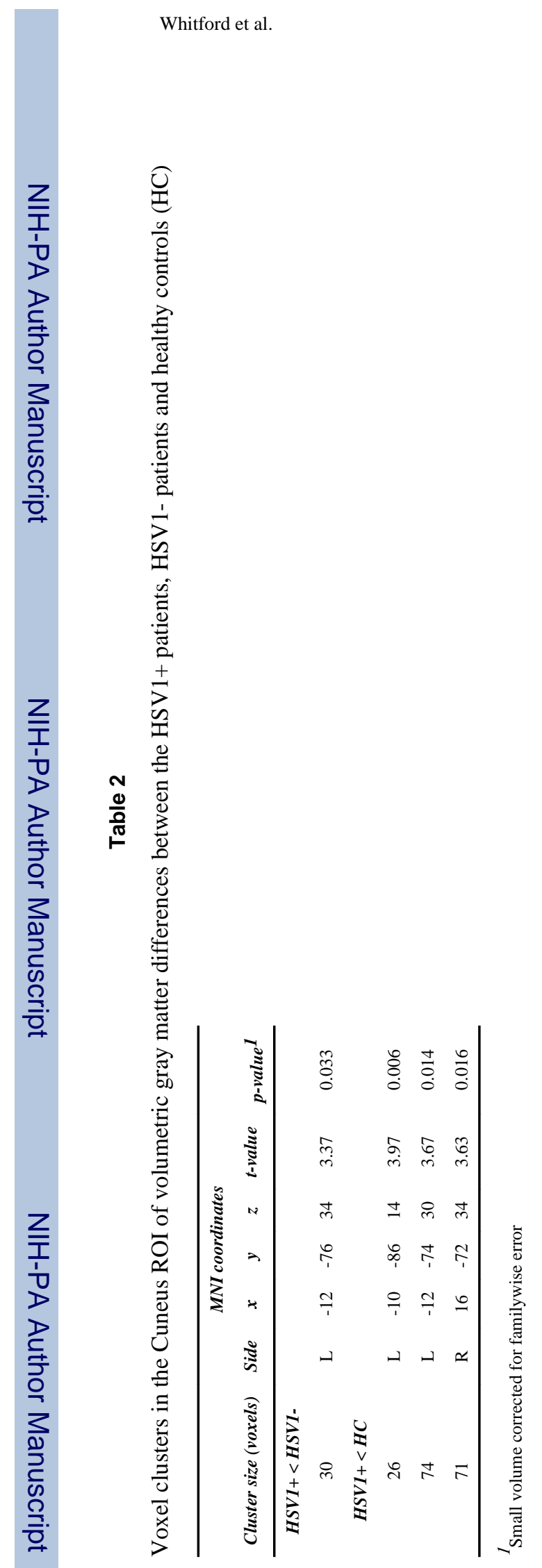

Schizophr Res. Author manuscript; available in PMC 2013 March 01. 